\title{
Bouncing off the Walls: The Influence of Gas-Kinetic and van der Waals Effects in Drop Impact
}

\author{
Mykyta V. Chubynsky, ${ }^{1, *}$ Kirill I. Belousov, ${ }^{2, \dagger}$ Duncan A. Lockerby, ${ }^{3, \ddagger}$ and James E. Sprittles $\circledast^{1, \oplus}$ \\ ${ }^{1}$ Mathematics Institute, University of Warwick, Coventry CV4 7AL, United Kingdom \\ ${ }^{2}$ Faculty of Physics and Engineering, ITMO University, St. Petersburg 197101, Russia \\ ${ }^{3}$ School of Engineering, University of Warwick, Coventry CV4 7AL, United Kingdom
}

(Received 19 June 2019; accepted 11 December 2019; published 26 February 2020)

\begin{abstract}
A model is developed for liquid drop impact on a solid surface that captures the thin film gas flow beneath the drop, even when the film's thickness is below the mean free path in the gas so that gas kinetic effects (GKE) are important. Simulation results agree with experiments, with the impact speed threshold between bouncing and wetting reproduced to within 5\%, while a model without GKE overpredicts this value by at least $50 \%$. To isolate GKE, the pressure dependence of the threshold is mapped and provides experimentally verifiable predictions. There are two principal modes of contact leading to wetting and both are associated with a van der Waals driven instability of the film.
\end{abstract}

DOI: 10.1103/PhysRevLett.124.084501

Liquid drop impact on solid surfaces [1] is of interest in situations ranging from inkjet printing $[2,3]$ to aeronautics $[4,5]$. It is also a complex, multistage, and multiscale process. Of all the phenomena associated with drop impact, splashing has received the most attention because of its complexity and the unexpected role of the ambient gas [6-10]. Experimental studies of the splashing mechanism have revealed that drops can "skate on a film of air" for an unexpected duration before touchdown [11,12], culminating in the discovery that drops can "bounce off walls" when contact is entirely prevented [13-15]. Recent experiments have revealed the strongly multiscale nature of this phenomenon: millimeter sized drops are suspended by air films four orders of magnitude smaller (i.e., by nanofilms) [12,16-20].

Despite experimental advances, the precise mechanism by which ambient gas pressure changes can alter splashing remains a topic of debate. The physical effects proposed for the gas film's behavior include (1) gas kinetic effects (GKE; also known as rarefied gas effects) [8,21-23], which become relevant for gas micro- and nanofilms, (2) inertia [24], and/or (3) compressibility [6,7], any of which can influence the stages of (i) precontact impact and (ii) postcontact wetting $[25,26]$. A full understanding of splashing would require all physics to be captured from the nano- to millimetric scale, with stage (i) providing an initial condition for (ii). Despite recent progress in this direction [27],

Published by the American Physical Society under the terms of the Creative Commons Attribution 4.0 International license. Further distribution of this work must maintain attribution to the author(s) and the published article's title, journal citation, and DOI. unambiguous conclusions remain elusive, so that isolating the specific physics at each stage remains an important route to further understanding.

This Letter focuses on the impact stage, where recent experimental analyses of drops bouncing off walls provide a perfect test bed for model verification. In particular, one would like to predict the transitions from drop bouncing to contact-induced wetting and, in doing so, address discrepancies between Kolinski et al.'s [13] experiments, where the air film height reaches $\sim 2 \mathrm{~nm}$ before contact, compared to de Ruiter et al.'s [14,15], where heights below 100$200 \mathrm{~nm}$ are never observed. Notably, there are similarities to the collision of droplets, where transitions between bouncing and coalescence are pressure dependent and have been captured by a computational model developed by $\mathrm{Li}$ [28] that incorporates GKE [29], and are further explored in [30]. Interestingly, there have been no equivalent studies, experimental or computational, investigating the pressure effect in the transition between bouncing and wetting for drops impacting solids. Li's theory is not applicable for this case, so in this Letter a more general computational model is developed to provide new physical insight and experimentally verifiable predictions for the impact phenomenon.

The drop impact problem is axisymmetric so that cylindrical coordinates $(r, z)$ are used (Fig. 1). We simulate water-glycerol drops with radius $R \sim 1 \mathrm{~mm}$, surface tension $\sigma$ and density $\rho_{l}$ similar to and viscosity $\sim 10$ times larger than that of water, as used in the experiments of Refs. $[13,14]$, in a broad range of ambient pressures $p_{0}$ to connect with previous experiments and make new predictions that highlight the physics associated with GKE. Since we are interested in skating and bouncing drops, especially near bouncing-wetting thresholds, we consider impact 


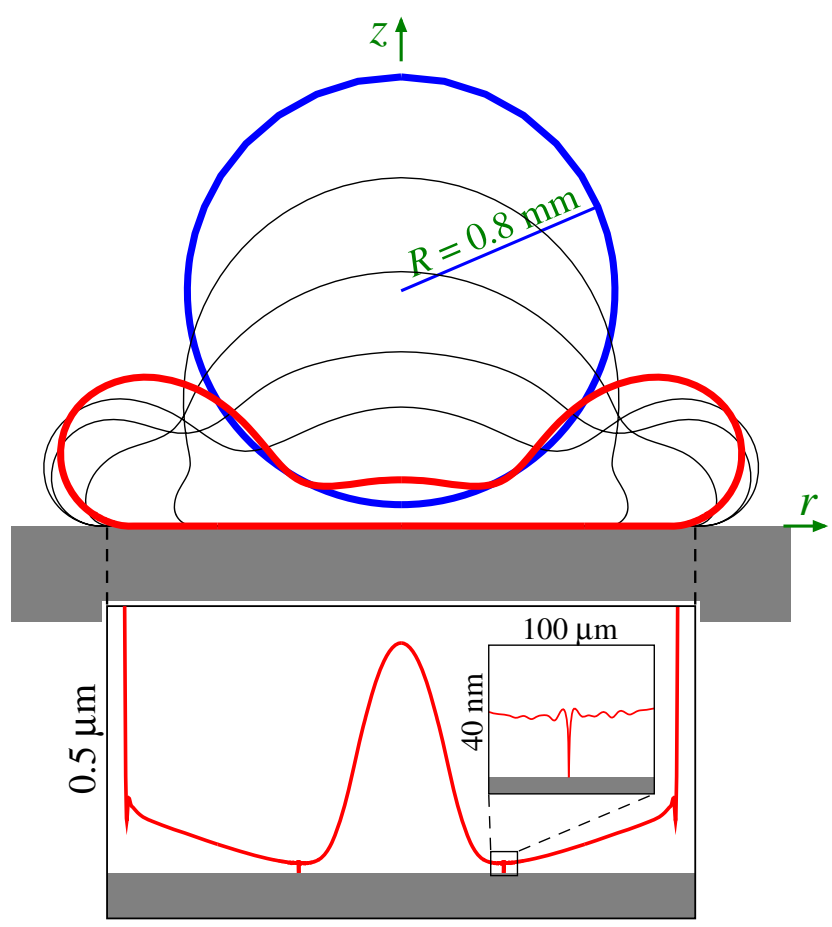

FIG. 1. The profiles of a water-glycerol drop (as described in the text) impacting a smooth solid surface at $0.76 \mathrm{~m} / \mathrm{s}$ at times $t=0$ (the initial condition; blue), $0.5,1,1.5,2$, and $\approx 2.6 \mathrm{~ms}$ (the moment of contact; red) obtained in our simulations. For the latter case, the profile of the bottom surface is shown separately, with the same scale in the horizontal direction, but magnified 2000 times in the vertical direction; an inset enlarges the vicinity of the contact.

speeds $V$ generally $\lesssim 1 \mathrm{~m} / \mathrm{s}$ so that inertia and compressibility in the gas are negligible and GKE are isolated, see Supplemental Material, Sec. I [31].

The restriction to moderate speeds allows us to make simplifying assumptions in the development of a lubrication model for the gas, which are justified in the Supplemental Material, Sec. I [31]. First, when far from the solid surface, the drop's deformation is negligible. Second, as the drop approaches the surface, significant drop deformation occurs only when the characteristic gap thickness $h \ll R$. Consequently, the drop's dynamics require only an accurate model for the air film where it is thin, where standard lubrication theory assumptions can be made [7,58-60].

The idea then is to solve the incompressible NavierStokes equations for the liquid drop with the air captured by lubrication theory and manifesting itself through the drop's boundary conditions. Specifically, the normal stress in the drop is assumed equal to the sum of the Laplace pressure and the gas pressure in the film $p_{g}(r, t)$ while the shear stress is equal to that in the gas next to the drop surface, $\tau_{g}(r, t)$. Away from the lubrication zone, the effect of the gas is negligible. To close the problem, $p_{g}(r, t)$ and $\tau_{g}(r, t)$ are determined by solving the lubrication problem for the air film.

For this lubrication problem, we need to consider an auxiliary problem of steady, incompressible and inertia-free gas flow in an infinite channel with parallel walls, one of which moves with respect to the other at a constant velocity $\mathbf{v}_{w}$ parallel to itself, in presence of a constant pressure gradient $\nabla p_{g}$. This flow is a superposition of a Couette flow component driven by the wall's motion with no pressure gradient, and the pressure-driven Poiseuille flow with immobile walls. Considering a planar surface of area $S$ spanning the channel and perpendicular to the walls, the mass flow rate $\Phi$ is the sum of the Couette and Poiseuille contributions,

$$
\begin{gathered}
\Phi=\Phi_{C}+\Phi_{P}, \\
\Phi_{C}=\frac{1}{2} S \rho_{g} \mathbf{v}_{w} \cdot \mathbf{n}_{S} \Delta_{C}^{\Phi}, \quad \Phi_{P}=-\frac{S \rho_{g} h^{2} \mathbf{n}_{S} \cdot \nabla p_{g}}{12 \mu_{g}} \Delta_{P}^{\Phi},
\end{gathered}
$$

where $\mathbf{n}_{S}$ is the normal to the surface $\mathrm{S}$ in the direction in which $\Phi$ is measured. These are the usual expressions for a continuum Stokes flow, except for the kinetic factors $\Delta_{C, P}^{\Phi}$, which are functions of the Knudsen number $\mathrm{Kn}=\lambda / h$, where $\lambda$ is the mean free path of the gas molecules and $h$ the channel height. These factors describe the influence of the GKE. Similarly, for the shear stress vector on the drop surface,

$$
\begin{gathered}
\boldsymbol{\tau}_{g}=\boldsymbol{\tau}_{C}+\boldsymbol{\tau}_{P}, \\
\boldsymbol{\tau}_{C}=\frac{\mu_{g} \mathbf{v}_{w} / h}{\Delta_{C}^{\tau}}, \quad \tau_{P}=\frac{\frac{1}{2} h \nabla p_{g}}{\Delta_{P}^{\tau}},
\end{gathered}
$$

which are classical lubrication theory expressions modified by kinetic factors $\Delta_{C, P}^{\tau}$. Equations (1)-(4) are valid for the full range of Knudsen numbers, from the Navier-Stokes limit $\mathrm{Kn} \rightarrow 0$ to the ballistic limit $\mathrm{Kn} \rightarrow \infty$; equivalent expressions are common in rarefied gas dynamics literature (see, e.g., [61,62]). By construction, all kinetic factors are unity $\left(\Delta_{C, P}^{\Phi, \tau}=1\right)$ at $\mathrm{Kn}=0$, where classical theory is valid. Henceforth, in the Letter we will make the simplest reasonable assumption that both the solid and the drop surfaces scatter gas molecules diffusely. Then by symmetry $\Delta_{C}^{\Phi}=\Delta_{P}^{\tau}=1$ (see the Supplemental Material, Sec. II [31]) and only two nontrivial kinetic factors remain, $\Delta_{C}^{\tau}$ and $\Delta_{P}^{\Phi}$. The consequences of allowing for partially specular reflection off the solid surface are considered in the Supplemental Material, Sec. V [31].

The Couette and Poiseuille flow problems for a rarefied gas in an infinite channel have been studied extensively by solving the kinetic Boltzmann equation and tabulated data from which $\Delta_{P}^{\Phi}$ and $\Delta_{C}^{\tau}$ can be deduced are available in the literature [63-67]. Based on this, interpolating formulae for 
$\Delta_{P}^{\Phi}$ and $\Delta_{C}^{\tau}$ have been obtained for use in our computations (see the Supplemental Material, Sec. II [31]). These formulae reproduce the literature data that span the range from very low to very high $\mathrm{Kn}$ to better than $2 \%$ and in addition preserve high- and low-Kn asymptotics and thus are expected to be very accurate for all Kn. Notably, even for $\mathrm{Kn}=1$, corresponding to approximately $100 \mathrm{~nm}$ air film heights, the kinetic factors are considerably higher than their classical value of unity $\left(\Delta_{C}^{\tau} \sim \Delta_{P}^{\Phi} \sim 10\right)$, so GKE are expected to be very significant during drop impact.

Equations (1)-(4) are the lubrication model for the gas film, which we assume remain valid locally even when the film thickness $h$ (and therefore $\mathrm{Kn}$ ) and the radial speed on the drop surface $v_{r}$ are functions of $r$ and $t$. Let $\Phi(r, t)$ be the mass flow rate through the cylindrical surface of radius $r$ spanning the air film. Then from mass conservation

$$
\frac{\partial \Phi}{\partial r}=-2 \pi r \rho_{g} \frac{\partial h}{\partial t}
$$

Using Eqs. (1) and (2), after some transformations we obtain

$$
\frac{\partial^{2} p_{g}}{\partial r^{2}}+g_{1}(r, t) \frac{\partial p_{g}}{\partial r}=g_{2}(r, t)
$$

where

$$
\begin{gathered}
g_{1}(r, t)=\frac{\partial}{\partial r}\left[\ln \left(r h^{3} \Delta_{P}^{\Phi}\right)\right], \\
g_{2}(r, t)=\frac{6 \mu_{g}}{r h^{3} \Delta_{P}^{\Phi}}\left[\frac{\partial}{\partial r}\left(r h v_{r}\right)+2 r \frac{\partial h}{\partial t}\right] .
\end{gathered}
$$

Equation (6) requires two boundary conditions which are taken to be

$$
\left.\frac{\partial p_{g}}{\partial r}\right|_{r=0}=0 ; \quad p_{g}\left(r=r_{0}\right)=p_{0},
$$

where $r_{0}$ is the boundary of the air film (see the Supplemental Material, Sec. III [31]). Equation (6) is solved numerically in parallel with the Navier-Stokes equations for the drop; from $p_{g}$, the shear stress at the boundary can be found using Eqs. (3) and (4).

A critical ingredient, discussed later, is van der Waals (vdW) interactions between the drop and the solid. As is standard in the literature $[28,29,68-70]$, these interactions are included by adding a disjoining pressure term

$$
p_{d}(r, t)=-\frac{A_{H}}{6 \pi h^{3}(r, t)},
$$

where $A_{H}$ is the Hamaker constant, to the normal stress condition on the drop boundary bordering the air film.
Our computational approach was implemented using COMSOL MULTIPHYSICS [71] and FREEFEM++ [72,73] finiteelement software (see the Supplemental Material, Sec. III [31]).

The computational model can also be applied, with minor modifications, to the head-on collision of two identical drops, where a plug flow replaces the Couette component so that only one nontrivial kinetic factor remains, $\Delta_{P}^{\Phi}$. If desired, this can be incorporated into an effective $\mu_{g}$ making it space and time dependent inside the gap, thus justifying Li's approach [28]. Therefore, we can benchmark our results against Li's, where the gas flow is computed in full, as well as experimental data, and excellent agreement is obtained at significantly reduced computational cost due to the lubrication approach (see the Supplemental Material, Sec. IV [31]). Notably, Li's approach fails for drop impact, where two kinetic factors are required.

To validate our approach for impact on a solid, we compare to experimental results by Kolinski et al. for $R=0.8 \mathrm{~mm}$ water-glycerine drops with kinematic viscosity $10 \mathrm{cSt}$ in $1 \mathrm{~atm}$ air [13] (exact parameters are in the Supplemental Material, Sec. I A [31]). These more viscous drops were considered instead of, say, the water drops studied extensively by de Ruiter et al. [15], to reduce the effect of drop oscillations prior to impact (see the Supplemental Material, Sec. I B [31]). A typical case is shown in Fig. 1.

First, we compare the results for the impact velocity threshold between bouncing without contact and contactinduced wetting. We find nearly perfect agreement, with the numerical threshold at $0.74 \mathrm{~m} / \mathrm{s}$ compared to $0.75 \mathrm{~m} / \mathrm{s}$ experimentally. For comparison, without $\operatorname{GKE}\left(\Delta_{C}^{\tau}=\right.$ $\Delta_{P}^{\Phi}=1$ ), at $1.1 \mathrm{~m} / \mathrm{s}$ contact has still not occurred (details below).

Second, we look at the evolution of the air film profile for a $0.55 \mathrm{~m} / \mathrm{s}$ impact. There is excellent qualitative agreement, with both a "dimple" on the axis and a "kink" near the edge reproduced (Fig. 2; see also a video comparison in the Supplemental Material [31]). One can see that both the impact duration and the maximum radius of the film are reproduced correctly; however, closer inspection shows that the film is thicker computationally, with the minimum height around $80 \mathrm{~nm}$ compared to about $50 \mathrm{~nm}$ experimentally. A likely explanation, given the excellent agreement for the wetting threshold, is a bias in the experimental TIR method used by Kolinski et al. (noted in Ref. [18]). The discrepancy in the lowest film height right before the wetting threshold (around $20 \mathrm{~nm}$ computationally vs only a few $\mathrm{nm}$ experimentally) is likely of the same nature.

We can now exploit our computational model to make new predictions that isolate GKE by considering the dependence on ambient pressure $p_{0}$. In Fig. 3 the minimum air film thickness as a function of time has been plotted, 

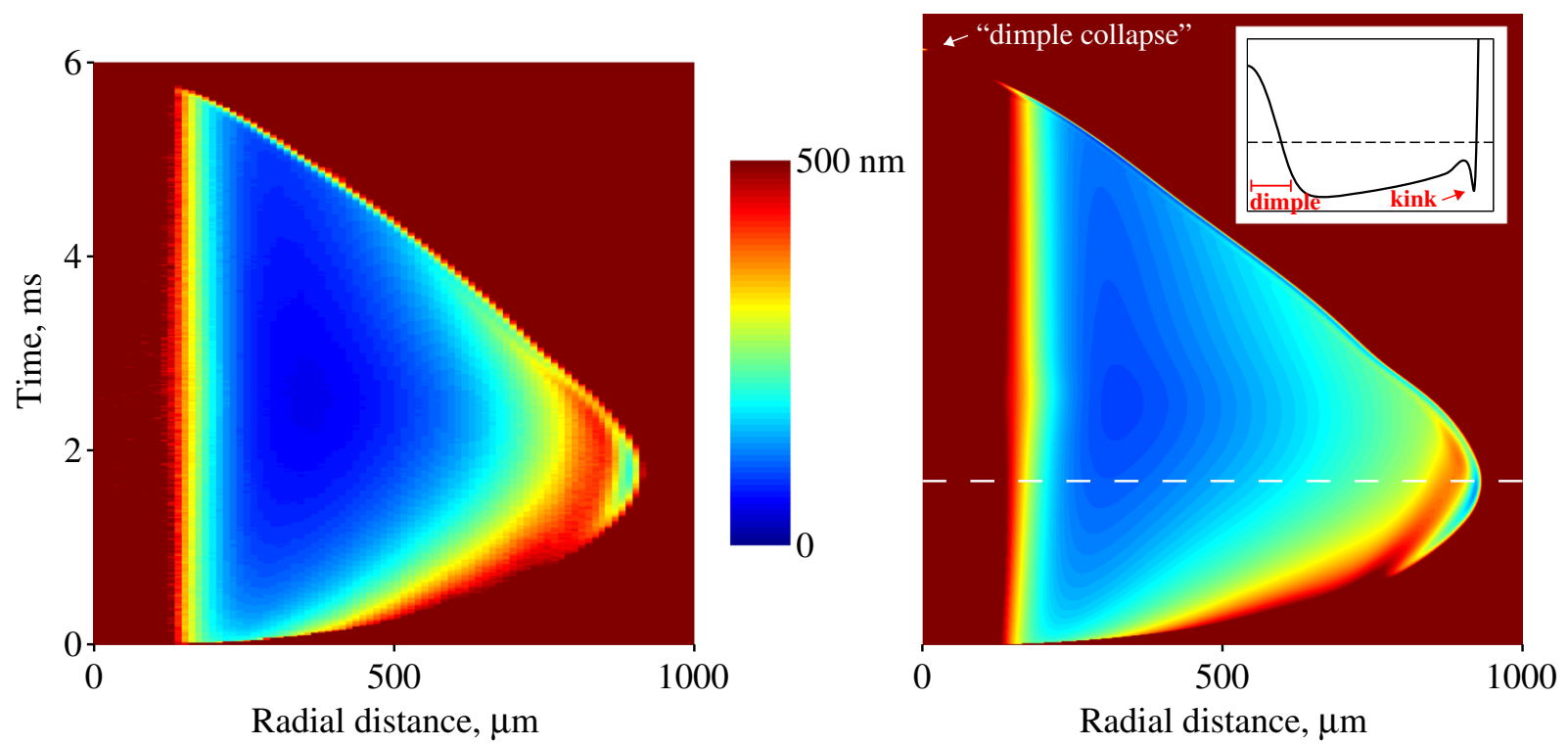

FIG. 2. Experimental (left) and computational (right) results for the time dependence of the air film thickness profile for drop impact with impact speed $V=0.55 \mathrm{~m} / \mathrm{s}$. The film thickness is indicated by the colorbar. The experimental colormap is replotted from Ref. [13]. The inset of the computational colormap shows the profile at the time of maximum spreading of the droplet (indicated by the white dashed line); the dashed line in the inset corresponds to the thickness of $500 \mathrm{~nm}$. "Dimple collapse" is discussed in the Supplemental Material, Sec. VII [31].

with different curves corresponding to the same impact speed $(0.4 \mathrm{~m} / \mathrm{s})$ but different $p_{0}$. This creates a transition between bouncing and wetting at a threshold pressure. In fact, a bouncing regime does not exist at all for $p_{0}<0.2$ atm (Fig. 4).

Interestingly, despite suggestions in the literature [74,75] that GKE alone are sufficient to induce contact, we have

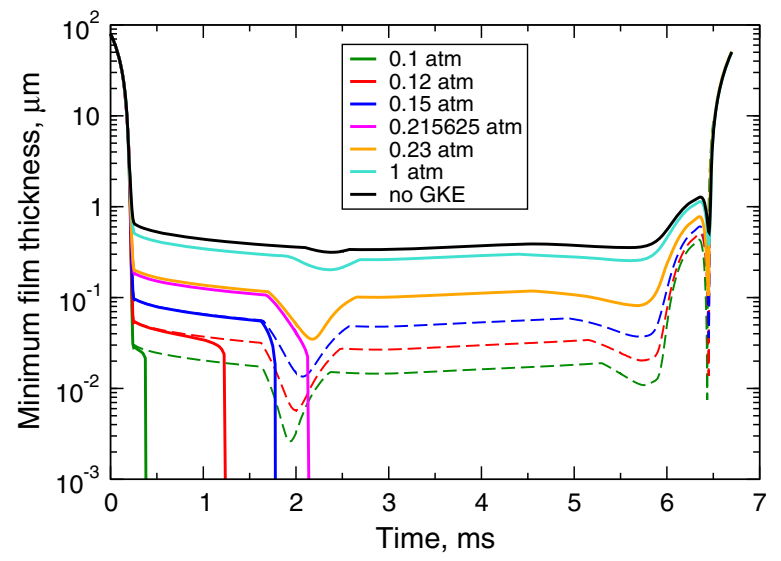

FIG. 3. Air film thickness at the thinnest point as a function of time for $V=0.4 \mathrm{~m} / \mathrm{s}$ and different ambient pressures, with (solid lines) and without (dashed lines for the three lowest pressures) $\mathrm{vdW}$ interactions. Dips around 2-2.5 ms are time intervals during which the film is thinner at the "kink" near the edge than in the flat part of the film. Starting with the lowest pressure, for the first two solid curves contact occurs before the dip (film mode of contact), for the next two curves it occurs at the kink during the dip (kink mode), and for the last three curves there is no contact (bouncing regime). Without vdW, there is never contact. observed there is never contact without vdW interactions (e.g., see Fig. 3 and the Supplemental Material, Sec. VI [31]). The regime diagram (Fig. 4) shows the existence of two different modes of contact, both driven by $\mathrm{vdW}$ forces, with first contact either at the kink (kink mode) or (as in Fig. 1) within the air film (film mode). These modes have been observed experimentally [15],

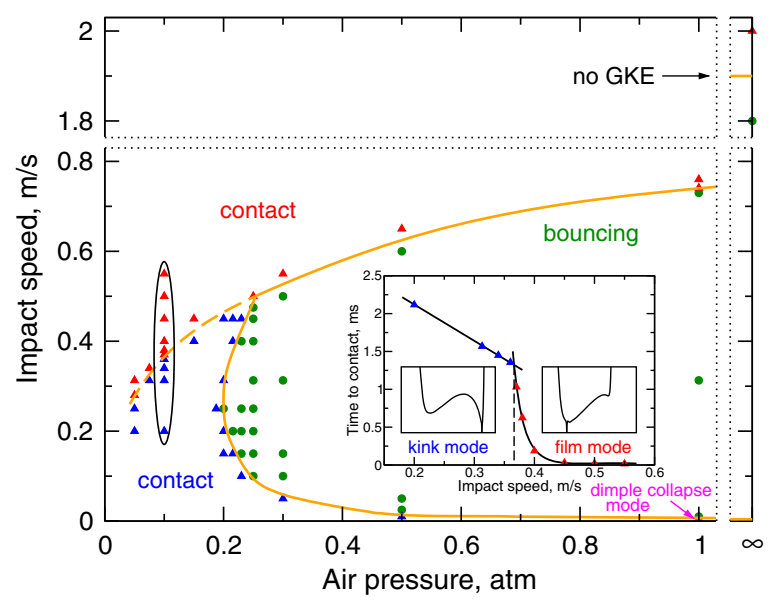

FIG. 4. Regime diagram with bouncing and contact (wetting) regimes and three different contact modes within the latter. The inset shows the time to contact (measured from the contact time for a drop in vacuum without vdW interactions), with the lines to guide the eye, and film thickness profiles at the time of contact in the two modes. The value of the threshold for $\mathrm{Kn} \rightarrow 0$ (formally corresponding to $p_{0} \rightarrow \infty$ and equivalent to no GKE) is approximate (see the Supplemental Material, Sec. VII [31]). 
but simulations reveal additional details, such as the characteristic wavelength of the instability, which is in agreement with linear instability theory (see the Supplemental Material, Sec. VI [31]). Such details would be missed by imposing a cut-off distance at which to initiate contact, as is often considered (see the Supplemental Material, Sec. VI [31]).

For both modes of contact, the instability is initiated when the distance from the surface is a few tens of nanometers, which is larger than recorded in experiments of Kolinski et al. [13]. On the contrary, in experiments of de Ruiter et al. [14], which use glass surfaces that are not atomically smooth, contact is initiated when the air film is hundreds of nanometers thick, likely due to surface defects. As pointed out by Kolinski et al. [13], even though the rootmean-squared roughness of these surfaces is on the order of $1 \mathrm{~nm}$, this is not an implausible explanation, since a single asperity penetrating the air film is sufficient for contact leading to wetting. The critical thickness will then vary from sample to sample depending on the statistical properties of the surface topography.

Notably, there is also a third "dimple collapse mode" (see Fig. 4) in which contact occurs at the drop axis just before the lift-off, existing at very low impact speeds $(<0.01 \mathrm{~m} / \mathrm{s}$ at $1 \mathrm{~atm})$. This mode is responsible for "bouncing drops" eventually wetting the surface and there is some very recent unpublished experimental evidence for its existence [76].

Promisingly, the threshold between the first two modes can be identified independently of the film profile: as the inset of Fig. 4 demonstrates, near to the transition in mode, a tiny change in the impact speed can yield a massive reduction in the time for the drop to wet the surface; this can be measured routinely using a side-on high speed camera.

In contrast to recent dynamic wetting results [26], GKE remain critical even at $1 \mathrm{~atm}$ as in their absence contact does not occur at $1.1 \mathrm{~m} / \mathrm{s}$ and is unlikely to until $2 \mathrm{~m} / \mathrm{s}$ (see the Supplemental Material, Sec. VII [31]). This is more than a factor of two higher than experiments and the GKE model predict. The large influence of GKE is due to significant decrease of both of the effective viscosities in the air film, which simultaneously reduces the pressure buildup (leading to a smaller film thickness) and speeds up the development of the instability leading to contact. Formally, eliminating GKE corresponds to $p_{0} \rightarrow \infty$ (see Fig. 4) while assuming that the gas remains ideal and the drop is not deformed when moving through air. For the experiments considered here, such conditions should be satisfied for pressures at about $10 \mathrm{~atm}$ (see the Supplemental Material, Sec. I [31]) where one should observe contact thresholds more than double that observed for $1 \mathrm{~atm}$.

The computational model can now be developed in complementary directions. Splashing can be tackled, where the main challenges are numerical, but many other recent experimental results also reveal unexpected flow structures that their authors claim are driven by GKE. Examples ripe for theoretical investigation include sawtooth contact line instabilities [77], double contacts upon impact [78], "extreme wetting" [79], and the Leidenfrost effect [80-82].

This work has been financially supported by EPSRC (UK) (Grants No. EP/N016602/1, No. EP/P020887/1, and No. EP/P031684/1) and the Leverhulme Trust.

*M.Chubynsky@warwick.ac.uk

†belousov_k.i@mail.ru

†D.Lockerby@warwick.ac.uk

JJ.E.Sprittles@warwick.ac.uk

[1] C. Josserand and S. T. Thoroddsen, Drop impact on a solid surface, Annu. Rev. Fluid Mech. 48, 365 (2016).

[2] B. Derby, Inkjet printing of functional and structural materials: Fluid property requirements, feature stability and resolution, Annu. Rev. Mater. Res. 40, 395 (2010).

[3] Fundamentals of Inkjet Printing: The Science of Inkjet and Droplets, edited by S. D. Hoath (Wiley-VCH, Weinheim, Germany, 2016).

[4] Z. Wu, Drop "impact" on an airfoil surface, Adv. Colloid Interface Sci. 256, 23 (2018).

[5] R. Cimpeanu and D. T. Papageorgiou, Three-dimensional high speed drop impact onto solid surfaces at arbitrary angles, Int. J. Multiphase Flow 107, 192 (2018).

[6] L. Xu, W. W. Zhang, and S. R. Nagel, Drop Splashing on a Dry Smooth Surface, Phys. Rev. Lett. 94, 184505 (2005).

[7] S. Mandre and M. P. Brenner, The mechanism of a splash on a dry solid surface, J. Fluid Mech. 690, 148 (2012).

[8] G. Riboux and J.M. Gordillo, Experiments of Drops Impacting a Smooth Solid Surface: A Model of the Critical Impact Speed for Drop Splashing, Phys. Rev. Lett. 113, 024507 (2014).

[9] Y. Liu, P. Tan, and L. Xu, Kelvin-Helmholtz instability in an ultrathin air film causes drop splashing on smooth surfaces, Proc. Natl. Acad. Sci. U.S.A. 112, 3280 (2015).

[10] A. M. P. Boelens and J. J. de Pablo, Simulations of splashing high and low viscosity droplets, Phys. Fluids 30, 072106 (2018)

[11] S. Mandre, M. Mani, and M. P. Brenner, Precursors to Splashing of Liquid Droplets on a Solid Surface, Phys. Rev. Lett. 102, 134502 (2009).

[12] J. M. Kolinski, S. M. Rubinstein, S. Mandre, M. P. Brenner, D. A. Weitz, and L. Mahadevan, Skating on a Film of Air: Drops Impacting on a Surface, Phys. Rev. Lett. 108, 074503 (2012)

[13] J. M. Kolinski, L. Mahadevan, and S. M. Rubinstein, Drops can bounce from perfectly hydrophilic surfaces, Europhys. Lett. 108, 24001 (2014).

[14] J. de Ruiter, R. Lagraauw, D. van den Ende, and F. Mugele, Wettability-independent bouncing on flat surfaces mediated by thin air films, Nat. Phys. 11, 48 (2015).

[15] J. de Ruiter, D. van den Ende, and F. Mugele, Air cushioning in droplet impact. II. Experimental characterization of the air film evolution, Phys. Fluids 27, 012105 (2015). 
[16] M. M. Driscoll and S. R. Nagel, Ultrafast Interference Imaging of Air in Splashing Dynamics, Phys. Rev. Lett. 107, 154502 (2011).

[17] J. de Ruiter, F. Mugele, and D. van den Ende, Air cushioning in droplet impact. I. Dynamics of thin films studied by dual wavelength reflection interference microscopy, Phys. Fluids 27, 012104 (2015).

[18] M. Shirota, M. A. J. van Limbeek, D. Lohse, and C. Sun, Measuring thin films using quantitative frustrated total internal reflection (FTIR), Eur. Phys. J. E 40, 54 (2017).

[19] K. R. Langley, E. Q. Li, and S. T. Thoroddsen, in Highspeed interferometry under impacting drops, in The MicroWorld Observed by Ultra High-Speed Cameras, edited by K. Tsuji (Springer, Cham, Switzerland, 2018), pp. 321-341.

[20] K. R. Langley and S. T. Thoroddsen, Gliding on a layer of air: Impact of a large-viscosity drop on a liquid film, J. Fluid Mech. 878, R2 (2019).

[21] L. Duchemin and C. Josserand, Rarefied gas correction for the bubble entrapment singularity in drop impacts, C. R. Mécan. 340, 797 (2012).

[22] C. S. Stevens, Scaling of the splash threshold for lowviscosity fluids, Europhys. Lett. 106, 24001 (2014).

[23] J. E. Sprittles, Air entrainment in dynamic wetting: Knudsen effects and the influence of ambient air pressure, J. Fluid Mech. 769, 444 (2015).

[24] Z. Jian, C. Josserand, S. Popinet, P. Ray, and S. Zaleski, Two mechanisms of droplet splashing on a solid substrate, J. Fluid Mech. 835, 1065 (2018).

[25] A. Marchand, T. S. Chan, J. H. Snoeijer, and B. Andreotti, Air Entrainment by Contact Lines of a Solid Plate Plunged into a Viscous Fluid, Phys. Rev. Lett. 108, 204501 (2012).

[26] J. E. Sprittles, Kinetic Effects in Dynamic Wetting, Phys. Rev. Lett. 118, 114502 (2017).

[27] A. M. P. Boelens, A. Latka, and J. J. de Pablo, Observation of the pressure effect in simulations of droplets splashing on a dry surface, Phys. Rev. Fluids 3, 063602 (2018).

[28] J. Li, Macroscopic Model for Head-on Binary Droplet Collisions in a Gaseous Medium, Phys. Rev. Lett. 117, 214502 (2016).

[29] P. Zhang and C. K. Law, An analysis of head-on droplet collision with large deformation in gaseous medium, Phys. Fluids 23, 042102 (2011).

[30] M. Liu and D. Bothe, Toward the predictive simulation of bouncing versus coalescence in binary droplet collisions, Acta Mech. 230, 623 (2019).

[31] See Supplemental Material at http://link.aps.org/ supplemental/10.1103/PhysRevLett.124.084501 for details, which includes Refs. [32-57].

[32] K. Takamura, H. Fischer, and N. R. Morrow, Physical properties of aqueous glycerol solutions, J. Pet. Sci. Eng. 98-99, 50 (2012).

[33] H. J. Butt, K. Graf, and M. Kappl, Physics and Chemistry of Interfaces (Wiley-VCH, Weinheim, Germany, 2003).

[34] H. Lamb, Hydrodynamics, 6th ed. (Cambridge University Press, Cambridge, England, 1932), pp. 639-641.

[35] A. Gopinath and D. L. Koch, Collision and rebound of small droplets in an incompressible continuum gas, J. Fluid Mech. 454, 145 (2002).
[36] C. Cercignani, M. Lampis, and S. Lorenzani, Plane Poiseuille flow with symmetric and nonsymmetric gas-wall interactions, Transp. Theory Stat. Phys. 33, 545 (2004).

[37] W.-L. Li, A database for interpolation of Poiseuille flow rate for arbitrary Knudsen number lubrication problems, J. Chin. Inst. Eng. 26, 455 (2003).

[38] W.-L. Li, A database for Couette flow rate considering the effects of non-symmetric molecular interactions, J. Tribol. 124, 869 (2002).

[39] J. C. Maxwell, On stresses in rarified gases arising from inequalities of temperature, Phil. Trans. R. Soc. London 170, 231 (1879).

[40] L. Wu and H. Struchtrup, Assessment and development of the gas kinetic boundary condition for the Boltzmann equation, J. Fluid Mech. 823, 511 (2017).

[41] M. D. Allen and O. G. Raabe, Re-evaluation of Millikan's oil drop data for the motion of small particles in air, J. Aerosol Sci. 13, 537 (1982).

[42] N. G. Hadjiconstantinou, The limits of Navier-Stokes theory and kinetic extensions for describing small-scale gaseous hydrodynamics, Phys. Fluids 18, 111301 (2006).

[43] F. Sharipov and V. Seleznev, Data on internal rarefied gas flows, J. Phys. Chem. Ref. Data 27, 657 (1998).

[44] S. Albertoni, C. Cercignani, and L. Gotusso, Numerical evaluation of the slip coefficient, Phys. Fluids 6, 993 (1963).

[45] S. K. Loyalka, Momentum and temperature-slip coefficients with arbitrary accommodation at the surface, J. Chem. Phys. 48, 5432 (1968).

[46] R. A. Cairncross, P. R. Schunk, T. A. Baer, R. R. Rao, and P. A. Sackinger, A finite element method for free surface flows of incompressible fluids in three dimensions. Part I. Boundary fitted mesh motion, Int. J. Numer. Methods Fluids 33, 375 (2000).

[47] I. Babuška and A. K. Aziz, Survey lectures on the mathematical foundations of the finite element method, in The Mathematical Foundations of the Finite Element Method with Applications to Partial Differential Equations, edited by A. K. Aziz (Academic Press, New York, 1972), pp. 5-359.

[48] K.-L. Pan, C. K. Law, and B. Zhou, Experimental and mechanistic description of merging and bouncing in headon binary droplet collision, J. Appl. Phys. 103, 064901 (2008).

[49] J. M. O. de Zárate and J. V. Sengers, Hydrodynamic Fluctuations in Fluids and Fluid Mixtures (Elsevier, Amsterdam, 2006).

[50] C. Zhao, J. E. Sprittles, and D. A. Lockerby, Revisiting the Rayleigh-Plateau instability for the nanoscale, J. Fluid Mech. 861, R3 (2019).

[51] A. Vrij, F. Th. Hesselink, J. Lucassen, and M. van den Tempel, Waves in thin liquid films. II. Symmetrical modes in very thin films and film rupture, Proc. K. Ned. Akad. Wet. Biol. Chem. Geol. Phys. Med. Sci. 73, 124 (1970).

[52] J. C. Padrino (private communication).

[53] J. M. Kolinski, The role of air in droplet impact on a smooth, solid surface, Ph.D. thesis, Harvard University, 2015.

[54] F. Brochard Wyart and J. Daillant, Drying of solids wetted by thin liquid films, Can. J. Phys. 68, 1084 (1990). 
[55] S. W. Sides, G. S. Grest, and M.-D. Lacasse, Capillary waves at liquid-vapor interfaces: A molecular dynamics simulation, Phys. Rev. E 60, 6708 (1999).

[56] L. R. Mason, G. W. Stevens, and D. J. E. Harvie, Multi-scale volume of fluid modelling of droplet coalescence, in The 9th International Conference on CFD in the Minerals and Process Industries, edited by C. B. Solnordal, P. Liovic, G. W. Delaney, and P. J. Witt (CSIRO, Melbourne, Australia, 2012), http://www.cfd.com.au/cfd_conf12/PDFs/179MAS .pdf.

[57] Y.-L. Hung, M.-J. Wang, J.-W. Huang, and S.-Y. Lin, A study on the impact velocity and drop size for the occurrence of entrapped air bubbles-water on parafilm, Exp. Therm. Fluid. Sci. 48, 102 (2013).

[58] B. J. Hamrock, S. R. Schmid, and B. O. Jacobson, Fundamentals of Fluid Film Lubrication, 2nd ed. (Marcel Dekker, New York, 2004).

[59] L. Duchemin and C. Josserand, Curvature singularity and film-skating during drop impact, Phys. Fluids 23, 091701 (2011).

[60] F. T. Smith, L. Li, and G. X. Wu, Air cushioning with a lubrication/inviscid balance, J. Fluid Mech. 482, 291 (2003).

[61] C. Cercignani, M. Lampis, and S. Lorenzani, Plane Poiseuille-Couette problem in micro-electro-mechanical systems applications with gas-rarefaction effects, Phys. Fluids 18, 087102 (2006).

[62] S. Fukui, H. Shimada, K. Yamane, and H. Matsuoka, Flying characteristics in the free molecular region (influence of accommodation coefficients), Microsyst. Technol. 11, 805 (2005).

[63] Y.W. Yap and J.E. Sader, High accuracy numerical solutions of the Boltzmann Bhatnagar-Gross-Krook equation for steady and oscillatory Couette flows, Phys. Fluids 24, 032004 (2012).

[64] L. B. Barichello, M. Camargo, P. Rodriguez, and C. E. Siewert, Unified solutions to classical flow problems based on the BGK model, Z. Angew. Math. Phys. 52, 517 (2001).

[65] W. Li, L.-S. Luo, and J. Shen, Accurate solution and approximations of the linearized BGK equation for steady Couette flow, Comput. Fluids 111, 18 (2015).
[66] C. Cercignani, M. Lampis, and S. Lorenzani, Variational approach to gas flows in microchannels, Phys. Fluids 16, 3426 (2004).

[67] C. Cercignani and C. D. Pagani, Variational approach to boundary-value problems in kinetic theory, Phys. Fluids 9, 1167 (1966).

[68] A. Vrij, Possible mechanism for the spontaneous rupture of thin, free liquid films, Discuss. Faraday Soc. 42, 23 (1966).

[69] A. Oron, S. H. Davis, and S. G. Bankoff, Long-scale evolution of thin liquid films, Rev. Mod. Phys. 69, 931 (1997).

[70] D. Bonn, J. Eggers, J. Indekeu, J. Meunier, and E. Rolley, Wetting and spreading, Rev. Mod. Phys. 81, 739 (2009).

[71] COMSOL Inc., www.comsol.com.

[72] F. Hecht, New development in FreeFem++, J. Numer. Math. 20, 251 (2012).

[73] www.freefem.org.

[74] M. H. Davis, Collisions of small cloud droplets: Gas kinetic effects, J. Atmos. Sci. 29, 911 (1972).

[75] G. A. Bach, D. L. Koch, and A. Gopinath, Coalescence and bouncing of small aerosol droplets, J. Fluid Mech. 518, 157 (2004).

[76] P. Chantelot and D. Lohse (private communication).

[77] M. Pack, P. Kaneelil, H. Kim, and Y. Sun, Contact line instability caused by air rim formation under nonsplashing droplets, Langmuir 34, 4962 (2018).

[78] E. Q. Li, K. R. Langley, Y. S. Tian, P. D. Hicks, and S. T. Thoroddsen, Double Contact During Drop Impact on a Solid Under Reduced Air Pressure, Phys. Rev. Lett. 119, 214502 (2017).

[79] K. Langley, E. Q. Li, and S. T. Thoroddsen, Impact of ultraviscous drops: Air-film gliding and extreme wetting, J. Fluid Mech. 813, 647 (2017).

[80] D. Quéré, Leidenfrost dynamics, Annu. Rev. Fluid Mech. 45, 197 (2013).

[81] E. Aursand, S. H. Davis, and T. Ytrehus, Thermocapillary instability as a mechanism for film boiling collapse, J. Fluid Mech. 852, 283 (2018).

[82] M. Shirota, M. A. J. van Limbeek, C. Sun, A. Prosperetti, and D. Lohse, Dynamic Leidenfrost Effect: Relevant Time and Length Scales, Phys. Rev. Lett. 116, 064501 (2016). 